IIntegrantes del Grupo de Estudios: Jaime Burrows (Facultad de Medicina, Universidad del Desarrollo), Carlos Echeverría B. (Presidente del Grupo de Estudios; Hospital Naval "Almirante Nef"), Alejandro Goic G. (Academia de Medicina del Instituto de Chile), Carolina Herrera C. (Clínica Avansalud) Carlos Quintana V. (Facultad de Medicina, Pontificia Universidad Católica de Chile), Alberto Rojas O. (Facultad de Medicina, Universidad Andrés Bello), Gonzalo Ruiz-Esquide (Clínica Santa María), Rodrigo Salinas R. (Facultad de Medicina, Universidad de Chile), Alejandro Serani M. (Facultad de Medicina, Universidad de los Andes), Paulina Taboada R. (Centro de Bioética, Pontificia Universidad Católica de Chile), Ricardo Vacarezza Y. (Facultad de Medicina, Universidad de Chile). Santiago de Chile.

Texto aprobado como Documento Oficial de la Sociedad Médica de Santiago-Sociedad Chilena de Medicina Interna, en sesión del Directorio, el 11 de marzo de 2014

Este trabajo no cuenta con grants ni financiamiento externo. Los autores declaran no tener conflictos de intereses. La Dra. Carolina Herrera C. informa que tiene un contrato de trabajo con el Laboratorio Novartis, Chile, donde se desempeña como Clinical Advisor Respiratory.

Correspondencia a:

Dr. Carlos Echeverría B. Hospital Naval "Almirante Nef". Subida Alessandri s/n. Viña del Mar. Chile. Teléfono: +5632 2573380 ciecheverria@hotmail.com

\section{El respeto a la intimidad del paciente}

\author{
GRUPO DE ESTUDIOS DE ÉTICA CLÍNICA \\ DE LA SOCIEDAD MÉDICA DE SANTIAGO ${ }^{1}$
}

\section{Respecting patient intimacy}

Transparency as a general rule for all our professional acts casts doubts about the statement of the Hippocratic Oath that says "Whatever I see or hear in the lives of my patients, I will keep secret, as considering all such things to be private". Medical secrecy protects the intimacy of patients, who reveal to their physicians their most hidden secrets aiming to recover their health. Therefore, physicians should receive those secrets with reverence and care, as servers and not as their owners. The values associated with the respect for personal intimacy are the anthropological basis of medical confidentiality. A medical act is performed by definition between two equally honorable individuals. Therefore, the professional honors the trust of his patient, maintaining strict confidence of what is revealed. Therefore, medical secrecy must be strengthened rather than weakened, pursuing common wealth and dignity.

(Rev Med Chile 2014; 142: 506-511)

Key words: Confidentiality; Hippocratic Oath; Personhood; Privacy.
"Lo que en el tratamiento, o incluso fuera de él, viere u oyere en relación con la vida de los hombres, aquello que jamás deba trascender, lo callaré teniéndolo por secreto".

\section{Juramento Hipocrático}

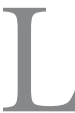
a complejidad y la despersonalización creciente de las interacciones en la civilización contemporánea, junto a la constatación dolorosa de la fragilidad humana en el ejercicio del poder, han hecho surgir en la cultura actual una reivindicación de 'transparencia' en el actuar social. Bajo esta exigencia, dinamizada por un deseo de justicia, se encuentra también un trasfondo de desconfianza hacia las personas y las instituciones sociales.

Por otra parte, el afán, de distintos estamentos de la sociedad, por supervisar y controlar el desem- peño de las acciones de los demás, bajo pretexto de optimizar los rendimientos económicos y políticos de lo ya realizado o por efectuar, está conduciendo a una carrera por el control de la información de las personas.

Frente a ello, la venerable tradición médica de la salvaguardia de la confidencialidad pareciera estar quedando fuera de época. ¿Cómo justificar una conducta basada en la confianza y la pretensión de sustraerse al control de quienes detentan el poder? Frente a estos desafíos se hace necesario reexaminar críticamente la vigencia del secreto médico, sus fundamentos, límites y la reserva de ciertos datos.

\section{Lo íntimo}

Probablemente, la indicación hipocrática de guardar silencio "acerca de aquello que jamás deba 
trascender", es el primer testimonio expreso de una precaución que los médicos vendrían ejercitando desde tiempos inmemoriales. Las afirmaciones del libro bíblico del Eclesiástico (27,17 ss.): “El que revela secretos pierde la confianza y no encontrará a un amigo..." testimonian que la valoración positiva de esta conducta no se limitaba, ya en ese entonces, al actuar profesional, sino que abarcaba a las relaciones humanas en general. Así, no es de extrañar la permanente consideración que, tanto la sociedad como la profesión médica, han tenido a través de los siglos hacia las prescripciones del Juramento Hipocrático.

Se ha sugerido que aquello que busca proteger la conducta del secreto médico sería, en definitiva, lo intimo. Siguiendo el argumento, ése no sería sino un aspecto de una conducta más amplia: el respeto a la intimidad ${ }^{2}$. La custodia de la intimidad -conducta propiamente humana- tiene cierta analogía con la conducta animal instintiva de protección de la integridad física y del territorio. Sin desconocer la conexión que tiene el cuidado de la intimidad con dimensiones subconscientes o instintivas, es patente que en el ser humano esta conducta adopta el carácter de una protección inteligente y libre de la vida, como del desarrollo social y personal.

Lo íntimo tiene que ver con aquello que, perteneciéndole en exclusiva a una persona o a un grupo, no puede ser usado o conocido por otro sin que le sea libremente permitido o concedido. Lo íntimo se vincula así con lo propio. Pero no todo lo propio es íntimo. Lo íntimo, por su naturaleza, está en mayor o menor grado oculto, y está relacionado estrechamente con el normal desenvolvimiento de la vida personal; es decir, con la vida consciente y libre ${ }^{3}$. Se reconocen tres niveles de lo íntimo: la intimidad territorial o espacial, la intimidad corporal y la intimidad psicológica o espiritual ${ }^{4}$. La acción médica incide en estos tres niveles; ya sea por medio de acciones concretas sobre el enfermo, por gestos y palabras, o por compromisos tácitos o explícitos que se dan en la relación profesional-paciente.

El respetuoso y delicado acceso a la intimidad espacial y corporal de los pacientes se pone a prueba tanto en la entrevista médica como en el examen físico y los procedimientos terapéuticos. Baste mencionar el acto de desvestirse parcial o totalmente, que los pacientes deberían realizar con plena libertad, con conocimiento del sentido de las acciones médicas que se van a efectuar, respetando las formas o códigos culturales que testimonian y protegen la intención estrictamente profesional de la interacción.

Así como es clara la necesidad del respeto de la intimidad corporal, que eventualmente se mantiene a recaudo con la ropa, también es evidente la necesidad de proteger la intimidad espiritual del consultante, que el profesional conoce a través de la confidencia. Se hace patente entonces la dimensión de la confianza concedida y de su reserva indispensable. A nadie escapa la decepción y dolor que causa la traición a la confianza otorgada, más allá del hecho que tenga o no consecuencias. La necesidad de la custodia de la intimidad propia y ajena aparece entonces como un hecho fácilmente intuible por todo miembro de una comunidad humana mínimamente personalizada. De hecho, mientras más elaborado es el carácter personal de las relaciones, más intensa es la valoración y más delicado el respeto de la intimidad propia y ajena.

\section{La persona y su intimidad}

En todo acto médico participan personas, cada una poseedora de un valor intrínseco y constitutivo, que le confiere dignidad por el sólo hecho de existir. El descubrimiento y la valoración de la realidad propiamente personal de la vida humana ha sido particularmente profundizado y progresivamente aplicado a la vida civilizada por la tradición cultural occidental, desde los primeros siglos de la era cristiana.Kant, en la época moderna, se hace intérprete de esta tradición, expresando que la persona tiene valor y no precio, que es un fin en sí misma y que no puede ser tomada como un mero medio ${ }^{5}$. El filósofo contemporáneo Robert Spaemann señala que el sujeto humano en virtud de su carácter personal no es "algo" sino "alguien"6, a la vez que todos los hombres son personas ${ }^{7}$. La peculiaridad del sujeto humano denotada por el concepto de persona puede también caracterizarse a través del concepto del 'yo'. El 'yo personal' no se concibe entonces como una experiencia psicológica sino como el fundamento de toda experiencia psicológica posible y como la raíz existencial del ser humano como viviente. Por ello el 'yo personal' o 'la persona' aparece como lo más interior o 'íntimo' del ser humano. Lo íntimo, como indica la etimología, apunta a aquella zona 
que es más interior o interna ${ }^{8}$, y lo más interno es en definitiva el carácter personal del viviente humano, su yo personal inteligente y libre. Es desde ese núcleo ontológico que se despliega la realidad espiritual de 'lo íntimo'.

Tan reservada es esa zona, que en el ser humano es de natural ocurrencia el pudor, que se extiende, como bien lo señala Choza ${ }^{9}$, y a diferencia de la mera vergüenza, más allá del ámbito corporal. El pudor, por ejemplo, inhibe la manifestación de emociones o circunstancias espirituales en privado o en público. Sólo se develan ciertas cosas o hechos a personas seleccionadas. El pudor, en expresión del mismo Choza, es: "el hábito y la tendencia a mantener la posesión de la propia intimidad desde la instancia más radical de la persona (el yo), y a mantener dicha intimidad en el estado de máxima perfección posible, con vistas a una entrega por la cual se trasciende la soledad y se autoperfecciona el sujeto"10.

La persona humana, dado este carácter interior y escondido, ha sido caracterizada con justa razón como un 'misterio' y, no pocas veces, para bien o para mal, una verdadera sorpresa, incluso para sí misma. Desde la madrugada del pensamiento occidental, llega aun hasta nosotros el eco de la admonición délfica: "conócete a ti mismo", tarea inacabable y fuente de sabiduría ${ }^{11}$. Siempre latente en el arte, la filosofía y en la experiencia cotidiana, está la búsqueda del ser humano por descubrirse él, personalmente, como tal. A la vez participante de la naturaleza y también ajeno a ella, de ninguna manera parece revelarse con tanta claridad a sí mismo como en la vivencia de la reflexión y la libertad. La persona se encuentra abierta a un horizonte interior en el que ve al universo completo y más ${ }^{12}$. De hecho, ocupan su mente cosas que aún no existen y que, sin embargo, son los motores de su acción. Cuando se accede a la vida personal, cuando se tiene aunque sea un grado mínimo de interioridad e intimidad, cada uno alcanza una cierta visión de sí mismo y de su vida, sea como dotada de sentido o carente de él, y con un cierto carácter de totalidad y normatividad ${ }^{13}$. Es como si el hombre fuese un viajero hacia un destino que se propone a él mismo, con finalidad auto-asignada. En esta posesión interior de una orientación vital, que es propia y definitoria de la persona, radica de algún modo su libertad y su dignidad. Se trata de un bien que, intangible e incomunicable, es a la vez lo más precioso que cada uno posee y atesora, y de lo cual se enorgullece o se avergüenza. Por conservar ese bien hay quienes sacrifican hasta la vida corporal y son dignos de alabanza. Aparecen como grandes en la historia aquellos que no someten su conciencia, además de mantener su mundo interior alejado del arbitrio injusto de terceros como, por ejemplo, el caso de Sócrates ${ }^{14}$.

Es precisamente a este mundo interior al que el paciente nos invita cuando nos confía su historia. Lo hace para que lo ayudemos a recuperar su salud, pero también para apoyar su corazón sufriente en alguien que lo comprenda, lo acepte, lo quiera, que no lo juzgue y lo aconseje para continuar construyendo el proyecto de su existencia. Es nuestro deber acercarnos con reverencia y cuidado a este verdadero misterio, nunca como dueños, siempre como servidores y amigos.

Una persona que consulta a un médico le informa de algunos hechos objetivos, algunos incluso observables por terceros, como, por ejemplo, un aumento de volumen o una mancha en una zona corporal expuesta. También comparte vivencias particulares de su ser íntimo. Para ello se requiere generar condiciones para una comunicación personal. La instancia deberá ser oportuna, respetuosa, atenta, generadora de confianza. Al compartir su intimidad el paciente no deja por ello de ser su dueño, por lo que el profesional no puede hacer uso de 'lo ajeno compartido' como si fuese propio.

El acceso a lo íntimo, a aquella realidad oculta que a veces hasta el mismo paciente desconoce, busca realizarse en un clima humano particular, al que Laín Entralgo designaba como "amistad médica" ${ }^{15}$ : situación de confianza que permite y hasta invita a la confidencia. Ésta trae aparejada la expectativa y el compromiso de guardar reserva.

\section{Alcance y límites del secreto médico}

El antiguo principio hipocrático de la confidencialidad profesional ha sido recogido de modo universal en los códigos de ética médica de los más diversos lugares y épocas. En la actualidad y sólo a título de ejemplo, se puede mencionar que en los Estados Unidos de Norteamérica ${ }^{16}$ y en Canadá ${ }^{17}$ la confidencialidad de los datos recogidos por el médico es sostenida en sus documentos deontológicos. La Asociación Médica Mundial, en la Declaración de Ginebra, desde su versión inicial en 1948 hasta sus revisiones actuales, señala que 
se deben guardar y respetar los secretos confiados al médico, incluso después del fallecimiento del paciente $^{18}$. En Chile $^{19}$ y Argentina ${ }^{20}$, también se explicita que es obligatorio, en las mismas condiciones, tal y como se norma en España ${ }^{21}$.

Junto a los valores ligados al respeto a la intimidad personal, que dan su base antropológica al secreto médico, existe también hoy una mayor conciencia acerca de los múltiples atentados posibles a la autonomía individual. Si bien es cierto que el paciente es una persona $y$, como tal, un ser libre, que toma la decisión de consultar, el entregar información sensible desequilibra el poder e influencia que el profesional tiene sobre el paciente, con el consecuente compromiso de su autonomía y libertad. Esta posibilidad se incrementa hoy en la atención institucionalizada, dados los múltiples actores sociales que, a varios títulos, exhiben cuestionables prerrogativas para acceder a la información médica. Si el acto médico implica responder a la confianza otorgada, manteniendo la reserva de lo confiado, aparece actualmente con claridad la necesidad, no de debilitar el secreto médico, sino más bien el de reforzarlo. Se da aquí lo que Altisent ${ }^{22}$ llama el "pacto de la relación clínica", en el cual se acuerda tácitamente que el paciente coloca algo que le es propio a disposición del profesional que lo atiende, entendiendo que guardará el secreto. No hacerlo traiciona la relación entre médico y paciente, ya que el primero necesitaba hacer saber y el segundo conocer, para que se dé la relación fecunda que sigue el camino buscado de la salud posible.

Cuando alguien, ajeno a la relación clínica, tiene acceso a determinada información entregada bajo reserva, sin la autorización previa de quien la dio, viola la confidencialidad. Beauchamp y Childress $^{23}$ invocan argumentos a favor de la confidencialidad desde dos puntos de vista: a) consecuencialista, donde se hace mención de la erosión de la confianza necesaria entre médico y paciente si se rompe la reserva. Admiten excepciones, como en el caso de ciertas enfermedades contagiosas, abuso infantil o heridas por arma de fuego, pero dejando en claro que hay consecuencias para la confianza del paciente en el médico. Llegan a establecer que un sistema adecuado se daría sólo si se hubieran tomado en cuenta todas las consecuencias posibles, minimizando la transgresión. Y, b) desde el punto de vista del derecho a la privacidad y autonomía, de manera que la información solamente se podría poner en conocimiento de otro, mediante decisión autónoma del confidente.

El secreto médico incluye tanto la naturaleza de la enfermedad como todas las circunstancias relacionadas, vale decir, todas aquellas que, de ser reveladas, puedan ocasionar cualquier tipo de perjuicio al paciente o sus relacionados, incluyendo lo que -sin haber sido explícitamente confiado al médico- haya sido conocido por éste en el ejercicio de sus funciones.

Un tema debatido es si acaso existen o no excepciones al secreto médico. Unos sostienen que existirían causales eximentes mientras que otros estiman que la mayor parte -sino todas- esas causales, hacen que en realidad no exista secreto médico, con lo que desaparecería la obligación de confidencialidad. La cuestión va más allá de lo semántico. Si existen circunstancias eximentes, deben darse criterios claros para determinarlas, a riesgo de caer en la arbitrariedad, el acomodo a las veleidades de la presión social o el sometimiento al poder administrativo, económico o político. $\mathrm{Y}$, si no existen, deben darse también criterios claros para dirimir en qué casos el secreto no se configura.

Las razones más frecuentemente invocadas para limitar la obligación de confidencialidad ${ }^{24,25}$ son:

1. Evitar el daño a un tercero identificado.

2. Razones de salud pública.

3. Por imperativo de la ley.

4. Por exigencia de organismos financiadores o controladores de la atención de salud.

5. Para impedir un daño al propio paciente.

6. Para que el profesional pueda defenderse de acusaciones injustas.

A pesar de la diversidad de causales invocadas existe consenso en el ámbito médico ${ }^{26,27}$ de que estas situaciones deberían ser excepcionales, y que la entrega de información debiese ser siempre la mínima y estrictamente necesaria para atender al requerimiento.

La situación de pacientes portadores de agentes infecciosos de transmisión sexual ha puesto a prueba la causal de daño a un tercero identificado. Existe hoy reticencia a poner los medios para revelar la situación de riesgo real en que se encuentran los contactos sexuales del portador de un agente infeccioso. Para esto se invocan razones de autonomía. No parece razonable, sin embargo, soste- 
ner una preeminencia absoluta de la autonomía, que pueda justificar el daño a terceros inocentes.

Las razones de salud pública, la autoridad de la ley y la exigencia de control burocrático se reducen todas en definitiva a razones de bien común. Sin embargo, una de las justificaciones más poderosas que se invocan para la salvaguardia de la confidencialidad es, justamente, el bien común. Dice el argumento que: si las personas supiesen que sus circunstancias personales pueden ser reveladas no consultarían, con lo cual se vería afectada la salud pública, la paz social y el ordenamiento racional de las acciones de salud. Ahora bien, estos son justamente los bienes que la salud pública, la ley y la burocracia intentan conseguir. Todo indica, en consecuencia, que se hace necesario alcanzar en estas materias un justo medio prudencial a través de un debate transparente y respetuoso, y no de la imposición autocrática de normativas que se introducen, de facto, por los que detentan el poder.

La razonabilidad de las causales que invocan el bien del enfermo y la defensa del médico se imponen de suyo, y muestran con claridad que la guarda de la confidencialidad exige reconocer un orden de subordinación en los principios morales que no exime de un esfuerzo personal y prudencial de articulación.

\section{Conclusión}

Nuevas épocas traen nuevos desafíos. La civilización contemporánea ha llegado a hacer fáciles y accesibles bienes que otrora no eran conseguibles en absoluto, o, cuando lo eran, sólo alcanzaban a unos pocos. Por otra parte, el aumento de la población y la mayor complejidad de las interacciones han determinado, por el contrario, que problemas que antaño eran de fácil expediente, se hayan hecho hoy en día, cada vez más enmarañados y engorrosos.

Hemos expuesto las razones por las cuales la venerable tradición médica de la confidencialidad ha sido, en nuestra época, puesta a prueba. La exigencia de transparencia, la desconfianza y el afán de muchos por inmiscuirse hasta en los niveles más íntimos de la personalidad, parecerían estar augurando la obsolescencia de esta noble tradición. La historia de la humanidad, la reflexión antropológica y ética, y los deseos más íntimos de las personas, muestran lo contrario. Nunca como hoy, parece más necesaria la defensa y promoción de la intimidad personal. Una época como la nuestra, afectada por la tendencia a la despersonalización, la anomia y la instrumentalización de los individuos al servicio de finalidades pragmáticas, requiere, más que nunca, una defensa de aquellos reductos más propios, genuinos y específicamente humanos de la existencia comunitaria. Hacerlo requiere de un esfuerzo de reflexión, lucidez, espíritu libre y crítico, pero también una voluntad de acción y compromiso.

\section{Referencias}

1. Hipócrates. Juramento. Biblioteca Gredos. Ed. Gredos, Madrid 2007. pág. 40.

2. Serani A, Respeto a la intimidad del paciente. Fundamentos antropológicos y éticos. En: Lavados M \& Serani A, "Ética Clínica, fundamentos y aplicaciones", Ediciones Universidad Católica (Santiago de Chile) 1994, II parte, cap. 5, pp. 103-20.

3. Cf. Ibid.

4. Ibid. p.104.

5. Kant I. Fundamentación para una Metafísica de las Costumbres. Alianza Editorial, Madrid. 2002.

6. Spaemann R. Personas: acerca de la distinción entre "algo" y “alguien”. EUNSA, Pamplona. 2000.

7. Spaemann R. ¿Todos los hombres son personas? RIALP, Madrid. 1992.

8. Real Academia Española de la Lengua, Diccionario de la Lengua Española 22a. Edición, Espasa, Madrid. España. 2001.

9. Choza J. La supresión del pudor, signo de nuestro tiempo y otros ensayos. EUNSA, Pamplona. 1990.

10. Ibid. 9, página 28.

11. Platón, Cármides. 165a. Ed. Gredos, Madrid. 1981.

12. Casanova Guerra C, El hombre: frontera entre lo inteligible y lo sensible. Ediciones de la Universidad Católica de Chile, Santiago de Chile. 2010.

13. Vigo A, Aristóteles. Una introducción. Instituto de Estudios de la Sociedad. Santiago de Chile. 2006, pp. 185-90.

14. Platón. Apología de Sócrates. 28 y y ss. Ed. Gredos, Madrid. 1981.

15. Laín Entralgo P, El médico y el enfermo. Triacastela. Madrid. 2003, Cap. 5, pp. 163-92.

16. American Medical Association. AMA's Code of Medical Ethics. Disponible en: http://www.ama-assn.org/ama/ pub/physician-resources/medical-ethics/code-medicalethics/opinion505.page? [Consultado el 5 de octubre de 2013]. 
17. Kleinman I, Baylis F, Rodgers S, Singer P. Bioethics for clinicians. Confidentiality. Can Med Assoc J 1997; 156 (4): 521-4.

18. Asociación Médica Mundial. Declaración de Ginebra. Disponible en: http://www.unav.es/cdb/ammginebra1. html [Consultado el 5 de octubre de 2013].

19. Colegio Médico de Chile. Código de Ética. Disponible en:http://www.colegiomedico.cl/Portals/0/files/ etica/120111codigo_de_etica.pdf [Consultado el 5 de octubre de 2013].

20. Confederación Médica de la República Argentina. Código de Ética. Disponible en: http://colmedse.com.ar/docs/ codigoetica.pdf [Consultado el 5 de octubre de 2013].

21. Consejo General de Colegios Oficiales de Médicos. Código de Deontología Médica. Madrid, España. Julio de 2011. Disponible en: https://www.cgcom.es/sites/default/files/codigo_deontologia_medica.pdf [Consultado el 5 de octubre de 2013].

22. Altisent R. Cuestiones éticas en el uso de la historia clínica. En: La Implantación de los derechos del paciente. P. León Sanz (Ed.) EUNSA, Barañain, España. 2004. pp.
308-25.

23. Beauchamp TL, Childress FC. Principles of Biomedical Ethics. Sixth Ed. Oxford University Press, New York. 2009. pp. 302-10.

24. República de Chile. Ley $\mathrm{n}^{\circ}$ 20.584. Sobre derechos y deberes que tienen las personas en relación con las acciones vinculadas a su atención en salud.

25. American Medical Association. Report of the Council on Ethical and Judicial Affairs. CEJA Report 4-I-06 Subject: Opinion E-5.05, "Confidentiality," Amendment Presented by: Robert M. Sade, MD, Chair. November 2006. Disponible en: http://www.ama-assn.org/resources/ doc/code-medical-ethics/505a.pdf [Consultado el 18 de diciembre de 2013].

26. Ferrer Colomer M. Secreto Profesional. Veracidad y Consentimiento Informado. Objeción de Conciencia. En: Manual de Bioética. G.M. Tomás G. (Coord.) Ariel S.A., Barcelona, España. 2001. pp. 128-39.

27. Cantero Rivas, R. La historia clínica: propiedad y acceso. En: La implantación de los derechos del paciente. P. León S. (Ed.). EUNSA, Pamplona. 2004. pp. 327-47. 